\title{
Low Vacuum Scanning Electron Microscopy, a Novel Technique for Integrated Microscopic Evaluation of Implantable Devices
}

\author{
F.J. Clubb, Jr. ${ }^{1}$, M.R. Coscio ${ }^{2}$, R. Nichols ${ }^{1}$, A. Rossi ${ }^{3}$, L. Stagnoli ${ }^{3}$, S. Sedlik ${ }^{3}$, E. Koullick ${ }^{2}$, M. \\ Bergan $^{2}$, E. Fogt ${ }^{2}$, M. Petersen ${ }^{4}$ and C.B. McClay ${ }^{4}$ \\ ${ }^{1}$ Department of Cardiovascular Pathology, Texas Heart Institute, Houston, TX 77225 \\ ${ }^{2}$ Materials and Biosciences Center, Medtronic, Minneapolis, MN 55432 \\ ${ }^{3}$ AVE, Medtronic, Santa Rosa, CA 95403 \\ ${ }^{4}$ Physiological Research Laboratories, Medtronic, Minneapolis, MN 55448
}

Scanning electron microscopy (SEM) is one of the tools available to the investigator for evaluating the topography of tissue/implantable device interfaces at higher magnification. Specimen preparation (i.e., critical point drying and sputter coating with a thin layer of gold/palladium) results in exclusion of the specimen for further evaluation by light microscopy (LM) or transmission electron microscopy (TEM). However, the development of low vacuum scanning electron microscopy (LVSEM) provides a means of direct correlation of tissue and device interaction of the same specimen/device. This paper will describe the advantages of combining LVSEM with LM and/or TEM.

Preparing the specimen is similar to that done routinely for LM or TEM. If both LM and TEM are required, a $2 \%$ paraformaldehyde $/ 2 \%$ glutaraldehyde fixative is used. After fixation, the specimen/device is dehydrated with graded ethyl alcohol. Once absolute alcohol concentration is reached, the specimen/device can be dried at room air or in a vacuum desiccator, which makes it ready for evaluation. The specimen can be mounted on an aluminum stub using double-sided tape, graphite glue, or copper tape. The specimen is then placed in a LVSEM (JSM-5900LV) and examined under low vacuum. For biologic specimens from stents or catheters the best settings are $10 \mathrm{Kv}$, Spot 40 and 18-40 $\mathrm{Pa}$ pressure. The lower pressure provides better resolution (Fig 1, endothelial covering of a vascular stent). However, with excessive charging from the device, then pressure is increased at the expense of resolution. LVSEM holds an important advantage over traditional SEM: after examining the specimen/device by LVSEM the specimen can still be evaluated with LM (Figs $2 \mathrm{a} \& 2 \mathrm{~b}$ ) and/or TEM (Fig 3). Moreover by using the Exakt ${ }^{\mathrm{TM}}$ plastic embedding and microgrinding system, direct correlation of LM sections can be made by TEM. Figure 3 is TEM section of the tissue in Fig $2 \mathrm{~b}$ (circled cell in Fig 2b). Thus, LVSEM, LM and TEM can be performed with the same specimen. Furthermore, LVSEM provides an excellent tool to help identify the presence of tissue in a catheter. Figures $4 \mathrm{a}-\mathrm{b}$ show neuropil in a catheter explanted from a rat brain. After identifying the location, tissue is removed and evaluated by TEM (Fig 4c). Another advantage of LVSEM is the topographic evaluation of the implantable device and correlating the device/tissue interface (Figs 5a-b). Since the LVSEM technique does not interfere with further evaluation by LM or TEM, the catheter and tract is bisected and then evaluated by LVSEM and LM (Figs 5c-d).

In conclusion, low vacuum scanning electron microscopy can be a powerful investigative technique in evaluation of implantable devices. We found that low vacuum scanning electron microscopy, when combined with light microscopy and transmission electron microscopy can reduce study cost and animals needed, and provide a more precise qualitative and quantitative evaluation of implantable devices. 

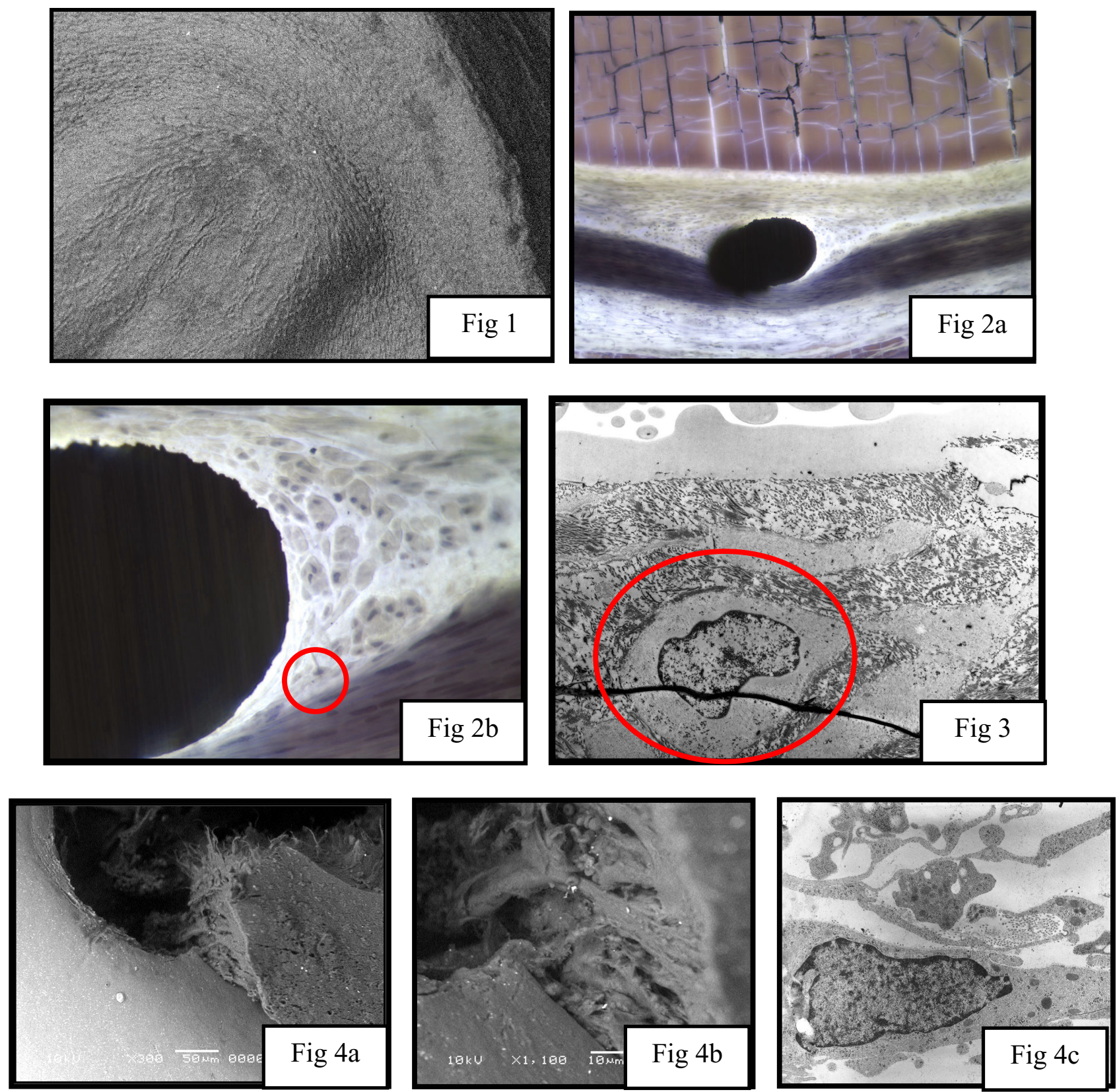

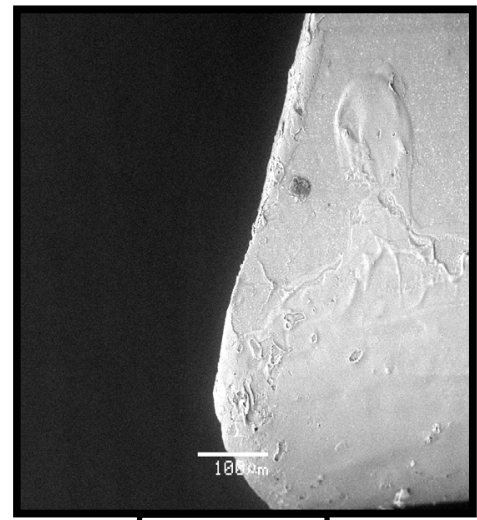

Fig $5 \mathrm{a}$
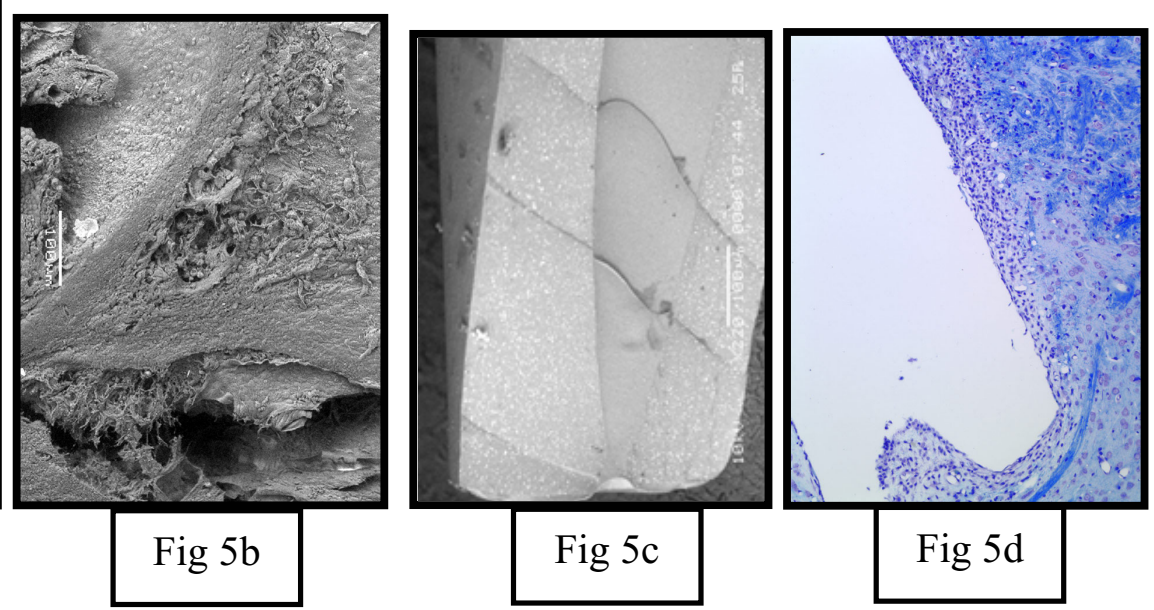